\title{
Effects of neoadjuvant chemotherapy combined with radiotherapy in patients with advanced esophageal carcinoma
}

\author{
YU LIU ${ }^{1,2}$, DEYAO XIE ${ }^{2}$, CHANG LI $^{1}$, CHAOXI LIN $^{2}$ and JUN ZHAO ${ }^{1}$ \\ ${ }^{1}$ Department of Thoracic Surgery, The First Affiliated Hospital of Soochow University, Medical College \\ of Soochow University, Suzhou, Jiangsu 215006; ${ }^{2}$ Department of Thoracic Surgery, The First Affiliated Hospital \\ of Wenzhou Medical University, Wenzhou, Zhejiang 325000, P.R. China
}

Received March 17, 2016; January 9, 2017

DOI: 10.3892/ol.2017.6515

\begin{abstract}
We analyzed the improvement of survival time and the effects of neoadjuvant chemotherapy combined with radiotherapy on treating patients with advanced esophageal carcinoma. Retrospectively, 43 patients were selected with esophageal carcinoma who were administered neoadjuvant chemotherapy combined with radiotherapy. According to gender, and tumor staging, the nearest neighbor matching was carried out. Eighty-six patients (1:2) who received neoadjuvant chemotherapy and 129 patients (1:3) who underwent surgery only were taken and compared for clinical outcomes. It was found that in the combination group, the median survival time was prolonged and the 1 -year survival rate improved. The diameter of tumors was significantly reduced, and the surgical resection, margin negative and total effective rates improved. In addition, the recurrence rate significantly decreased, whereas quality of life scores significantly increased $(\mathrm{p}<0.05)$. The comparison of overall incidence of complications was not statistically significant ( $p>0.05$ ). Tumor staging, location, and diameter after neoadjuvant therapy, as well as therapeutic regimen, treatment cycle, margin negative rate and effective rate were independent risk factors for significantly influencing survival outcomes and time $(\mathrm{p}<0.05)$. In conclusion, neoadjuvant chemotherapy combined with radiotherapy can be utilized to treat advanced esophageal carcinoma improve survival time and promote prognosis.
\end{abstract}

\section{Introduction}

Esophageal carcinoma is a common malignant tumor that ranks 8th in the world and 4th in China. In China, there are

Correspondence to: Dr Jun Zhao, Department of Thoracic Surgery, The First Affiliated Hospital of Soochow University, Medical College of Soochow University, 188 Shizi Street, Suzhou, Jiangsu 215006, P.R. China

E-mail: zhaojia0327@126.com

Key words: advanced esophageal carcinoma, neoadjuvant chemotherapy combined with radiotherapy, nearest neighbor matching, median survival time, quality of life approximately 259,000 new cases annually, and the number of deaths are 211,000 cases every year which accounts for more than $50 \%$ of cases globally (1). The pathological type was mainly squamous cell carcinoma, and most patients had arrived at the advanced stage before diagnosis. Surgical resection mostly occurs in stage I to IIA; nevertheless, the local recurrence rate reaches $40-60 \%$ and the 5-year overall survival rate was only $30 \%$ (2).

To the best of our knowledge, there is currently no standard treatment paradigm for esophageal carcinoma in stage IIB to IV. Conventional radiotherapy and chemotherapy after operation were palliative therapies, while radiotherapy and chemotherapy prior to surgery (or neoadjuvant therapy) may improve the surgical resection rate, survival time and reduce the recurrence rate (3). However, only a few studies have been conducted on a small scale using different radiotherapy and chemotherapy regimens, yielding inconsistent results, which bring great challenges for clinical treatments $(4,5)$. At the same time, screening of the groups benefiting mostly is also difficult. According to retrospective conclusion, this study examined improvement of survival time and the effects of neoadjuvant chemotherapy combined with radiotherapy on treating patients with advanced esophageal carcinoma.

\section{Patients and methods}

Patients. We retrospectively summarized patients diagnosed with esophageal carcinoma (squamous cell carcinoma) in the First Affiliated Hospital of Soochow University from January, 2012 to January, 2015. Inclusion criteria for patients were, age 18-70, functional status, and KPS scores $>69$. It was the first diagnosis, initial treatment for the selected patients. The patients met the 2002 6th edition international standard of TNM staging of esophageal carcinoma (stage IIB to IV), excluding esophageal fistula and viscera metastasis. The blood biochemistry was WBC $\geq 4.0 \times 10^{9} / 1$, PLT $\geq 100 \times 10^{9} / 1$ and $\mathrm{HGB} \geq 100 \mathrm{~g} / \mathrm{l}$, with normal functions of liver and kidney and complete clinical data. Exclusion standards were patients with thoracic injury, surgical history and other diseases that need to combine with radiotherapy, chemotherapy or surgery to treat, patients with tracheoesophageal fistula confirmed by fiberoptic bronchoscope or upper digestive tract radiography, patients with diseases combined with second primary 
Table I. Comparison of baseline information in three groups.

\begin{tabular}{lccccccccc}
\hline Group & Cases & Male/female & Age (years) & IIB & III & IV & Upper thoracic & Middle & Lower \\
\hline Combination & 43 & $25 / 18$ & $42.3 \pm 6.7$ & 19 & 17 & 7 & 6 & 20 & 17 \\
Simple & 86 & $48 / 38$ & $43.5 \pm 6.6$ & 36 & 36 & 14 & 12 & 42 & 32 \\
Surgical & 129 & $78 / 51$ & $44.2 \pm 6.5$ & 60 & 51 & 18 & 13 & 62 & 54 \\
F-value $\left(\chi^{2}\right)$ & & 0.463 & 0.524 & & 0.560 & & & 1.120 & \\
P-value & & 0.793 & 0.821 & & 0.967 & & & 0.891 \\
\hline
\end{tabular}

malignant tumor (excluding patients who had recovered from skin and cervical cancer in situ over 5 years), patients with diseases combined with major underlying diseases who did not tolerate radiotherapy and chemotherapy at a certain dose, patients withdrawn from treatments, and cases with poor compliance. This study was approved by the Ethics Committee of our hospital and written informed consent was obtained of the patients or their families.

Finally 43 patients were selected who were administered neoadjuvant chemotherapy combined with radiotherapy, designated as the combination group. According to gender and tumor staging, the nearest neighbor matching was carried out, 86 patients (1:2) were selected who received neoadjuvant chemotherapy as the simple (standard) group and 129 patients (1:3) who underwent surgery as the surgical group. Baseline information is shown in Table I.

Treatment methods. In the combination group, there were 28 cases that received neoadjuvant chemotherapy, with $60-80 \mathrm{mg} / \mathrm{m}^{2}$ of cisplatin (DDP) and $500-1,000 \mathrm{mg} / \mathrm{m}^{2}$ of 5-fluorouracil (5-FU) added from day 1 to 5. The days 21-28 were taken as a cycle, to perform 2 to 3 cycles. There were 6 cases with $80 \mathrm{mg} / \mathrm{m}^{2}$ of nedaplatin (NDP), $200 \mathrm{mg} /$ day of calcium folinate $(\mathrm{CF})$ added from day 1 to 5 and 1,000 $\mathrm{mg} /$ day of tegafur (FT)-207 added from day 1 to 5 . The 21st day was taken as a cycle to perform 2 cycles. There were 3 cases of ECF regimen (pharmorubicin + DDP + 5-FU), 3 cases with paclitaxel and DDP or carboplatin and 3 cases of MIC regimen (mitomycin + ifosfamide + DDP).

Neoadjuvant radiotherapy was used to evaluate tolerance during chemotherapy. Radiotherapy was employed at the same time or chemotherapy interval or after chemotherapy and BJ6B-400 6MV X was used as the linear accelerator by Elekta North Institute. There were 30 cases of three dimensional conformal radiation for 2 to 3 coplanar fields and 13 cases of emphatic radiation. Two anterior oblique portal plus wedges were used for cervical segment, and one anterior and two posterior fields for middle-lower, with 2 Gy each time, once every day and 5 times every week. The dosage range was 45-70 Gy/6 weeks, and the median dose was $60 \mathrm{~Gy} / 6$ weeks.

In the simple group, there were 60 cases with DDP and 5-FU, 12 cases with NDP, CF and FT-207, 5 cases with ECF, 7 cases with MIC and 2 other cases. Surgical methods were surgical resection or palliative therapy of conventional open surgery.

The differences among median survival time, 1-year survival rate, average biggest diameter of tumors, surgical

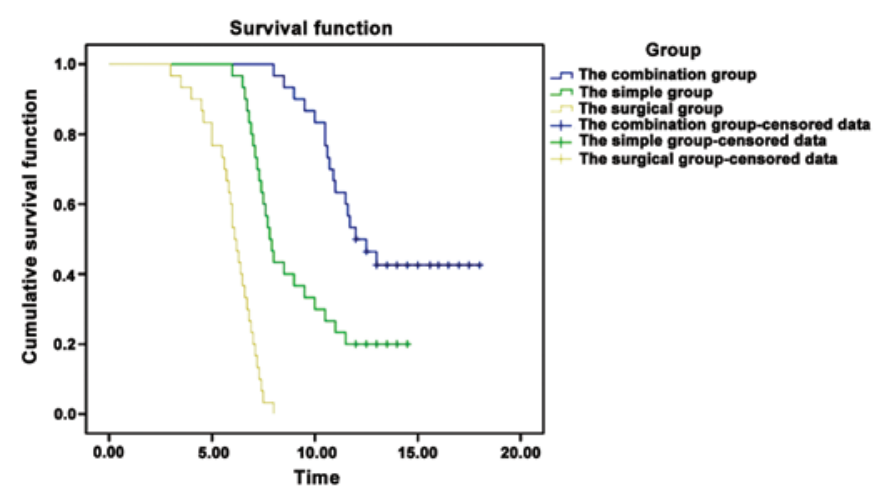

Figure 1. Kaplan-Meier (KM) method for survival time.

resection rate, margin negative rate, effective rate, recurrence rate, quality of life (QOL) scores and the incidence of complications related to neoadjuvant therapy, and screening of factors that may influence survival outcomes were compared. The CT scan was conducted for the measurement of tumor diameters, pathological examinations of tumors for margin and NCI solid tumor's effect evaluation criterion (RECIST 200 edition) for the effective rate. The recurrence rate included recurrence in situ and distant metastasis. Through the linear formula, the original score of each item in QLQ-c30 scale was converted into 0-100. QOL score change by 5-10 was considered as slight, 10-20 as obvious, and $>20$ as significant. The higher overall QOL scores were associated with better quality of life. According to common standards of toxicity of chemotherapy CTC 3.0 version and RTOCG grading standards of acute radiation injury, the incidence of complications included severe hematological complications (the increase of neutrophils, anaemia, hemorrhage, bone marrow transplantation), digestive and urinary systems (severe injuries of liver and kidney), and radiation esophagitis. On the basis of complication conditions, the symptomatic treatment was given or evaluated whether or not there was need to stop treatment.

Statistical analyses. According to sequence, the EpiData 3.1 data management software was used to input clinical data of every selected patients, with two copies for two individuals independently. SPSS 20.0 (Chicago, IL, USA) was used to analyze and deal with data, and the mean \pm standard deviation (SD) was used to indicate quantitative data. One-way ANOVA was used for comparison of several groups, and the independent sample t-test was employed for comparisons between two groups. The cases or percentage was used to indi- 
Table II. Comparison of survival time and survival rate.

\begin{tabular}{lccc}
\hline Group & $\begin{array}{c}\text { Median survival time } \\
\text { (months) }\end{array}$ & 95\% CI & 1-year survival rate \\
\hline Combination $(\mathrm{n}=43)$ & 12.0 & $10.224-13.776$ & $23(53.5 \%)$ \\
Simple $(\mathrm{n}=86)$ & 7.8 & $7.263-8.337$ & $35(40.7 \%)$ \\
Surgical $(\mathrm{n}=129)$ & 6.1 & $5.671-6.529$ & $32(24.8 \%)$ \\
$\chi^{2}$ & 94.079 & & 13.600 \\
P-value & $<0.001$ & & $<0.001$
\end{tabular}

The combination group, neoadjuvant chemotherapy combined with radiotherapy; the simple group, neoadjuvant chemotherapy.

Table III. Comparison of the diameter of tumors, the surgical resection rate and the margin negative rate.

\begin{tabular}{lcccc}
\hline Group & $\begin{array}{c}\text { Diameter of tumors } \\
\text { before treatment }(\mathrm{cm})\end{array}$ & $\begin{array}{c}\text { Diameter of tumors } \\
\text { after treatment }\end{array}$ & $\begin{array}{c}\text { Surgical resection } \\
\text { rate }\end{array}$ & $\begin{array}{c}\text { Margin negative } \\
\text { rate }\end{array}$ \\
\hline Combination $(\mathrm{n}=43)$ & $5.6 \pm 1.3$ & $3.3 \pm 0.9$ & $36(83.7)$ & $25(69.4)$ \\
Simple $(\mathrm{n}=86)$ & $5.4 \pm 1.2$ & $4.2 \pm 1.2$ & $60(69.8)$ & $30(50.0)$ \\
Surgical $(\mathrm{n}=129)$ & $5.5 \pm 1.4$ & - & $53(41.1)$ & $19(35.8)$ \\
F-value $\left(\chi^{2}\right)$ & 0.625 & $5.748^{\mathrm{a}}$ & 31.660 & 9.683 \\
P-value & 0.432 & 0.016 & $<0.001$ & 0.008 \\
\hline
\end{tabular}

${ }^{\mathrm{a}}$ t-test.

Table IV. Comparison of effective rate and the recurrence rate.

\begin{tabular}{|c|c|c|c|c|c|c|}
\hline Group & $\begin{array}{l}\text { Complete } \\
\text { remission }\end{array}$ & $\begin{array}{l}\text { Partial } \\
\text { remission }\end{array}$ & $\begin{array}{c}\text { Total } \\
\text { effective }\end{array}$ & $\begin{array}{l}\text { Local } \\
\text { recurrence }\end{array}$ & $\begin{array}{c}\text { Metastasis } \\
\text { rate }\end{array}$ & $\begin{array}{c}\text { Total recurrence } \\
\text { rate }\end{array}$ \\
\hline Combination $(n=43)$ & 17 & 20 & $37(86.0 \%)$ & 15 & 9 & $24(55.8 \%)$ \\
\hline Simple $(n=86)$ & 22 & 38 & $60(69.8 \%)$ & 30 & 26 & $56(65.1 \%)$ \\
\hline Surgical $(n=129)$ & 25 & 46 & $71(55.0 \%)$ & 44 & 59 & $103(79.8 \%)$ \\
\hline$\chi^{2}$ & & & 14.879 & & & 11.147 \\
\hline P-value & & & $<0.001$ & & & 0.004 \\
\hline
\end{tabular}

cate qualitative data, and the $\chi^{2}$ test was used for comparisons within the group. The Kaplan-Meier (KM) method (log-rank test) was used for median survival time, and Cox's proportional hazards regression model (forward method) was used for multifactor analysis. A difference of $\mathrm{p}<0.05$ was considered as statistically significant.

\section{Results}

Comparison of survival time and survival rate. In the combination group, median survival time significantly prolonged, and the 1-year survival rate improved significantly $(\mathrm{p}<0.05)$, as shown in Table II and Fig. 1.

Comparison of tumors diameter, surgical resection rate and margin negative rate. In the combination group, the diameter of tumors reduced significantly, whereas the surgical resection
Table V. Comparison of QOL scores and the incidence of complications.

\begin{tabular}{lcc}
\hline Group & QOL scores & $\begin{array}{c}\text { Incidence of } \\
\text { complications }\end{array}$ \\
\hline Combination $(\mathrm{n}=43)$ & $82.4 \pm 10.3$ & $5(11.6)$ \\
Simple $(\mathrm{n}=86)$ & $75.3 \pm 12.4$ & $7(8.1)$ \\
Surgical $(\mathrm{n}=129)$ & $62.6 \pm 13.5$ & - \\
F-value $\left(\chi^{2}\right)$ & 6.527 & 0.413 \\
P-value & $<0.001$ & 0.520
\end{tabular}

QOL, quality of life.

and margin negative rates improved significantly $(\mathrm{p}<0.05)$, as shown in Table III. 
Table VI. Analysis of Cox's proportional hazard regression model.

\begin{tabular}{lcrrrr}
\hline Factors & $\beta$ & Wald & P-value & RR & $95 \%$ CI \\
\hline Tumor staging & 0.125 & 10.425 & $<0.001$ & 3.953 & $2.320-5.203$ \\
Tumor location & 0.323 & 6.635 & $<0.001$ & 1.524 & $0.867-2.326$ \\
The diameter of tumors & 0.426 & 9.567 & $<0.001$ & 2.746 & $1.867-3.402$ \\
after treatment & & & & & \\
Therapeutic regimens & 0.627 & 12.524 & $<0.001$ & 4.527 & $3.654-5.133$ \\
Treatment cycle & 0.824 & 5.926 & 0.013 & 1.935 & $1.130-2.534$ \\
The margin negative rate & 0.329 & 11.425 & $<0.001$ & 3.236 & $2.935-4.531$ \\
The effective rate & 0.565 & 12.203 & $<0.001$ & 2.568 & $2.132-3.439$ \\
\hline
\end{tabular}

Comparison of effective rate and recurrence rate. In the combination group, the total effective rate improved significantly, and the recurrence rate decreased significantly $(\mathrm{p}<0.05)$, as shown in Table IV.

Comparison of $Q O L$ scores and the incidence of complications. In the combination group, QOL scores improved significantly $(\mathrm{p}<0.05)$. There were 4 cases that had complications related to chemotherapy and 1 case that had complications related to radiotherapy. The comparison of total incidence of complications was not statistically significant $(\mathrm{p}>0.05)$ as shown in Table V.

Analysis of Cox's proportional hazard regression model. The gender, age, tumor staging, tumor location, the diameter of tumors (before or after neoadjuvant therapy), therapeutic regimens (combination, simple, surgical), treatment cycle, surgical resection rate, margin negative rate, effective rate, recurrence rate, QOL scores and the incidence of complications as arguments, and survival outcomes and time as dependent variable, were included them in the model (Table VI).

\section{Discussion}

The theoretical advantages of neoadjuvant chemotherapy lie in (6) decrease tumor stage, reduce tumor volume, and increase surgical resection rate; control and treatment of micro-metastases and decrease in the recurrence rate; chemotherapeutics can reach tumor cells sufficiently passing undamaged blood supply systems; evaluate in vivo sensitivity to guide treatments after surgery. The disadvantages may be postponement of surgical time of patients with resectable esophageal cancer, thus missing the most suitable surgical timing (7). The advantages of neoadjuvant radiation includes accurate radiation, increase surgical time of patients with esophageal cancer, increase sensitivity of chemotherapeutic agents, increase dosage and chemotherapy cycle of chemotherapeutics and increase surgical resection rate, without enlarging damage of tissues around the tumors (8). The disadvantages were that radiation dose and regimens were inconsistent which can influence treatment outcomes (9).

A randomized study test reported by British Medical Research Council (MRC) included 802 cases of patients with resectable esophageal cancer, 400 cases in the neoadjuvant chemotherapy with 2 cycles of DDP and 5-FU and surgical treatments following, and 402 cases with simple surgery. The results showed that in the neoadjuvant chemotherapy group, both median survival time and 2-year survival rate improved, as reported (10). In another study, meta-analysis for randomized tests with 11 neoadjuvant chemotherapy or radiation, and the results manifested that in the neoadjuvant therapy group, the 2-year survival rate was higher than that in the simple surgical group, and it increased by $4.4 \%$ (95\% CI, 0.3-8.5) (11).

Another study included 440 cases with esophageal cancer, which were randomly divided into the neoadjuvant chemotherapy combined with surgery group and the simple surgical group (5). The results showed that in the combination group, the margin positive rate of surgery decreased significantly ( $4 \%$ vs. $15 \%, \mathrm{p}<0.01)$, but there were no obvious differences among median time of these two (14.9 vs. 16.1 months, $\mathrm{p}=0.53$ ), 1, 2 and 3-year survival rate ( 59 vs. $60 \%, 35$ vs. $37 \%, 23$ vs. $26 \%$ ) disease-free survival rate, or the post-operative complication rate. In another randomized trial, 2,051 patients with esophageal cancer obtained neoadjuvant chemotherapy combined with surgery group, there were no differences among 1 and 2-year survival rate, 3 and 4-year survival rate had a tendency to improve, and 5-year survival rate improved obviously $(\mathrm{RR}=1.44,95 \%$ $\mathrm{CI}=1.05-1.97, \mathrm{p}=0.02$ ) while comparing with the simple surgical group (12). Total surgical resection rate and pathological resection rate in these two groups were the same.

The differences of study results may be related to the number of samples, pathological types included in the groups (the ratio of adenocarcinoma or squamous cell carcinoma), chemotherapy regimens (DDP and 5-FU as basic regimens, also with pharmorubicin, bleomycin, methotrexate, paclitaxel, fluconazole, ifosfamide and other new drugs), and chemotherapy dose.

Neoadjuvant chemotherapy combined with radiation has synergistic reactions, and many clinical studies also suggest that neoadjuvant chemotherapy and radiation has more benefits for patients with esophageal cancer compared with simple surgical treatments. In a clinical non-controlled study with 69 cases of patients with esophageal cancer in the stage II (13), all the patients received chemotherapy with paclitaxel and DDP and hyperfraction radiotherapy synchronizing twice/day (1.5 Gy once). The results manifested that median survival time was 24 months, and 1,2 and 3-year 
survival rate was 75,50 and $34 \%$, respectively, which all increased. A meta-analysis included 9 randomized controlled trial with 1,116 patients, which suggested that neoadjuvant chemotherapy combined with radiation improves surgical resection rate, which reached $21 \%$ complete remission and decreased the incidence rate of local positions (14). Although the 1- and 2-year survival rate in the neoadjuvant chemotherapy combined with radiation group was similar to those in the simple surgical group, the 3-year survival rate improved. It also suggested that synchronous neoadjuvant chemotherapy and radiation before operation has more benefits than sequential chemoradiotherapy.

We found that in the combination group, median survival rate prolonged, 1-year survival rate improved, the diameter of tumors reduced, the surgical resection rate and the margin negative rate improved, total effective rate improved, the recurrence rate decreased, and QOL scores improved significantly. Comparatively, the total incidence of complications was not statistically significant. Independent risk factors that influence survival outcomes and time include tumor stage, tumor location, the diameter of tumors after neoadjuvant therapy, therapeutic regimens, treatment cycle, the margin negative rate and effective rate. This study is more close to real scenario, pathological type was squamous cell carcinoma patients in the stage IIB to IV have low surgical resection rate. Doctors and patients had more willingness to utilize neoadjuvant therapy, compliance of treatment was high, the regimens were more personal (more regimens and dose of chemotherapy and radiation can be selected), and follow-up was strict and information was complete. According to Cox's proportional hazards regression model, and screening for risk factors may influence survival outcomes.

In conclusion, employing neoadjuvant chemotherapy combined with radiation can be used to treat advanced esophageal cancer and can improve the median survival time and 1-year survival rate, improve surgical resection rate and promote prognostic survival.

\section{References}

1. Jemal A, Bray F, Center MM, Ferlay J, Ward E and Forman D: Global cancer statistics. CA Cancer J Clin 61: 69-90, 2011.

2. Ando N, Iizuka T, Ide H, Ishida K, Shinoda M, Nishimaki T, Takiyama W, Watanabe H, Isono K, Aoyama N, et al; Japan Clinical Oncology Group: Surgery plus chemotherapy compared with surgery alone for localized squamous cell carcinoma of the thoracic esophagus: a Japan Clinical Oncology Group Study - JCOG9204. J Clin Oncol 21: 4592-4596, 2003.
3. Boonstra JJ, Kok TC, Wijnhoven BP, van Heijl M, van Berge Henegouwen MI, Ten Kate FJ, Siersema PD, Dinjens WN, van Lanschot JJ, Tilanus HW, et al: Chemotherapy followed by surgery versus surgery alone in patients with resectable oesophageal squamous cell carcinoma: long-term results of a randomized controlled trial. BMC Cancer 11: 181, 2011.

4. Medical Research Council Oesophageal Cancer Working Group: Surgical resection with or without preoperative chemotherapy in oesophageal cancer: a randomised controlled trial. Lancet 359: 1727-1733, 2002.

5. Kelsen DP, Ginsberg R, Pajak TF, Sheahan DG, Gunderson L, Mortimer J, Estes N, Haller DG, Ajani J, Kocha W, et al: Chemotherapy followed by surgery compared with surgery alone for localized esophageal cancer. N Engl J Med 339: 1979-1984, 1998.

6. Lv J, Cao XF, Zhu B, Ji L, Tao L and Wang DD: Long-term efficacy of perioperative chemoradiotherapy on esophageal squamous cell carcinoma. World J Gastroenterol 16: 1649-1654, 2010.

7. Metzger R, Bollschweiler E, Drebber U, Mönig SP, Schröder W, Alakus H, Kocher M, Baldus SE and Hölscher AH: Neoadjuvant chemoradiotherapy for esophageal cancer: impact on extracapsular lymph node involvement. World J Gastroenterol 16: 1986-1992, 2010.

8. Campbell NP and Villaflor VM: Neoadjuvant treatment of esophageal cancer. World J Gastroenterol 16: 3793-3803, 2010.

9. Ajani JA, Barthel JS, Bentrem DJ, D'Amico TA, Das P, Denlinger CS, Fuchs CS, Gerdes H, Glasgow RE, Hayman JA, et al; National Comprehensive Cancer Network: Esophageal and esophagogastric junction cancers. J Natl Compr Canc Netw 9: 830-887, 2011.

10. Cunningham D, Allum WH, Stenning SP, Thompson JN, Van de Velde CJ, Nicolson M, Scarffe JH, Lofts FJ, Falk SJ, Iveson TJ, et al; MAGIC Trial Participants: Perioperative chemotherapy versus surgery alone for resectable gastroesophageal cancer. N Engl J Med 355: 11-20, 2006.

11. Kaklamanos IG, Walker GR, Ferry K, Franceschi D and Livingstone AS: Neoadjuvant treatment for resectable cancer of the esophagus and the gastroesophageal junction: a meta-analysis of randomized clinical trials. Ann Surg Oncol 10: 754-761, 2003.

12. Malthaner R and Fenlon D: Preoperative chemotherapy for resectable thoracic esophageal cancer. Cochrane Database Syst Rev: CD001556, 2003

13. Urba SG, Orringer MB, Ianettonni M, Hayman JA and Satoru H: Concurrent cisplatin, paclitaxel, and radiotherapy as preoperative treatment for patients with locoregional esophageal carcinoma. Cancer 98: 2177-2183, 2003.

14. Urschel JD and Vasan H: A meta-analysis of randomized controlled trials that compared neoadjuvant chemoradiation and surgery to surgery alone for resectable esophageal cancer. Am J Surg 185: 538-543, 2003. 